\title{
Acción colectiva y protesta en Venezuela. Dificultades teóricas y metodológicas en su estudio
}

\author{
(Collective action and protests in Venezuela. \\ Theoretical and methodological difficulties in their \\ study)
}

\author{
*Yorelis J. Acosta \\ Universidad Central de Venezuela, Caracas - Venezuela \\ *Email de correspondencia: yorelisaco@gmail.com
}

\section{RESUMEN}

Las transformaciones vividas en Venezuela en los últimos años han dado origen a protestas y acciones colectivas a lo largo del país, captando la atención de investigadores del área, quienes han abordado la acción colectiva, en su mayoría, desde un ángulo teórico. Partiendo de una revisión argumentativa de las principales aproximaciones teóricas sobre la temática, este trabajo pretende definir los elementos comunes de la acción colectiva y protestas en Venezuela para que, además de su utilidad en el planteamiento teórico, estos elementos puedan servir de guía en su planteamiento metodológico. Considerando estos elementos, en este trabajo se proponen directrices que permitan un mejor registro de los eventos a fin de disminuir los vacíos encontrados en las fuentes de datos de Venezuela. Los lineamientos propuestos tienen su inicio en la revisión y análisis de archivos periodísticos de prensa sobre las protestas, seguidos de la recopilación de testimonios de los actores involucrados, y una descripción de los eventos que incluya detalles como la identificación del evento, información sobre los actores de la protesta, acción, demandas, identidad, variables de orden político, y la articulación de los hechos y actores

Palabras clave: acción colectiva; protestas; dificultades metodológicas; Venezuela.

\section{ABSTRACT}

The changes experienced in Venezuela in recent years have led to protests and collective actions across the country, capturing the attention of researchers in this field, who have mostly addressed this collective action from a theoretical angle. Based on an argumentative review of the main theoretical approaches to the issue, this work aims at identifying common elements of collective action and 
protests in Venezuela so that, in addition to its usability in the theoretical approach, these elements can serve as a guide in its methodological design. Considering these elements, this article proposes guidelines that will allow a better record of events in order to reduce the gaps found in the data sources of Venezuela. The proposed guidelines have initiated in the review and analysis of news archives about the protests, followed by the collection of testimonies of the people involved, and a description of events that includes details such as the identification of the event, information about protest actors, action, demands, identity, political variables, and the articulation of the incidents and actors.

Keywords: collective action; protests; methodological difficulties; Venezuela. 


\section{INTRODUCCION}

Los años recientes han estado marcados en Venezuela por un proceso de transformación sociopolítica que se tradujo también en un mayor movimiento de la sociedad. Estas transformaciones sin duda, crearon las condiciones para la aparición de nuevos actores, acciones colectivas y protestas novedosas que coexisten con algunas condiciones del viejo orden y sus actores. Las demandas giran alrededor de estos cambios y necesidades no satisfechas de larga data, cuyo destinatario principal es el Estado, tanto en sus entes nacionales como regionales.

En nuestro país el proceso de movilización social ha tomado rasgos particulares, como, por ejemplo: ser un instrumento de medición de la fuerza política o del apoyo popular de los principales actores políticos del país, responder a la dinámica polarizada con dos grupos principales de actividad política, unos los seguidores del presidente Chávez y otros, quienes lo adversan, y en ocasiones los grupos se movilizan en sentidos contrarios ante una misma demanda.

Estas acciones de rechazo o de apoyo ante políticas públicas, de protestas y contra protestas, por darle un nombre, hacen más complejo el análisis de la acción colectiva y confunden los registros que se hacen en el país sobre acciones colectivas y protestas. Por otra parte, hay cambios en este fenómeno que requieren un nuevo tratamiento analítico que dé cuenta de esa nueva realidad.

La variedad y las consecuencias de las acciones colectivas, se evidencia no solamente en las calles y en el caos que genera en la ciudad, sino también en los canales que se usan para transmitir sus mensajes, como el Internet y los medios de comunicación social en especial la prensa y la TV utilizados para realizar el conteo del fenómeno. Así se van inaugurando nuevas acciones y su presencia es de mayor alcance, a la vez que se van articulando nuevas relaciones de poder en el marco territorial, político, cultural y económico donde se desenvuelve.

Por tanto, el aumento de las protestas y la acción colectiva ocupa un lugar central en la escena política del país y como fenómeno social debe llamar la atención a los estudiosos del área. Por otro lado, resulta de interés investigativo abordar el tema a partir de las propuestas teóricas predominantes y nuevas hipótesis explicativas y dimensiones definitorias de la acción colectiva que den cuenta del fenómeno y contribuya a su abordaje empírico, superando las limitaciones enfrentadas hasta ahora en los trabajos que intentan explicarlo.

Si bien las diversas teorías brindan herramientas útiles para el estudio de las acciones colectivas y las protestas, representan formas alternativas de entender el fenómeno, poniendo énfasis en distintas variables y tratan de responder a diferentes incógnitas de estas. Por tanto, me propongo en primer lugar, una revisión de las principales aproximaciones teóricas para definir los elementos comunes que puedan servir de guía para el abordaje teórico y metodológico de las protestas en Venezuela.

A diferencias de los trabajos teóricos, hay menos trabajos sobre aspectos metodológicos, que ofrecen herramientas limitadas en el abordaje del problema de la protesta en Venezuela que requiere de discusión.

En nuestro país, los trabajos de Margarita López Maya (2003, 2004, 2006), Luis Salamanca (2004) y María Urreiztieta (2008) dan cuenta de diferentes aproximaciones e intentos de comprensión de la acción colectiva. Sin embargo, los trabajos están plagados de dudas y sugerencias para superar algunas de las debilidades que se afrontan. Una de ellas, aspectos metodológicos relacionados con la definición y sus dimensiones de registros, el cual constituye otro de los objetivos del presente trabajo.

Los aspectos relacionados con el registro de las protestas, se abordan a partir de 1996 en el Cendes, quien, bajo la dirección de López Maya, inicia una investigación sobre la protesta popular venezolana elaborando un registro de las acciones colectivas en un periodo histórico que abarcó desde Gómez a la fecha, además de generar un marco interpretativo general sobre la acción colectiva. En estos trabajos logran construir la base de datos Bravo Pueblo, que se ha utilizado 
con frecuencia en investigaciones sobre el tema.

Por otra parte, el Programa Venezolano de Educación-Acción en Derechos Humanos (PROVEA) es otra organización que se ha dedicado a realizar una base de datos sobre las acciones de protestas, de hecho, la mayoría de las investigaciones realizadas en el país utilizan estos datos como principal fuente.

Ahora bien, el problema es mucho más complejo que el simple conteo de la presencia del evento, sus actores y demandas. A las ya tradicionales protestas, se han sumado en los últimos años un conjunto de actores y características novedosas, que van desde acciones episódicas-disruptivas hasta hechos violentos y acciones articuladas de más largo alcance, una de ellas conducente al debilitamiento del orden constitucional como los hechos acontecidos el 11,12 y 13 de abril de 2002 que sacaron al Presidente Hugo Chávez del poder momentáneamente.

Es en este horizonte, que el trabajo se propone identificar las dimensiones básicas de la acción colectiva a partir de los distintos planteamientos teóricos, incluyendo otra dimensión que le es consustancial: el abordaje metodológico. Así, constituye el eje central de este trabajo definir las dimensiones básicas de la acción colectiva, específicamente de las protestas y generar lineamientos que permitan un mejor registro de los eventos, a fin de superar las grandes dificultades encontradas en las datas disponibles en el país. Por tanto, la constante referencia a nuestro contexto es fundamental, ya que se pretende contribuir con el trabajo realizado por organizaciones no gubernamentales y académicas en relación al tema.

La tesis central es el reconocimiento del carácter multidimensional de la acción colectiva y la complementariedad de aspectos que deben analizarse para su conocimiento, tales como el origen de las acciones colectivas y las movilizaciones sociales, identificación de sus actores, formas de acción, la lógica de acción, la forma organizativa adquirida y el impacto de las acciones, así como describir la forma como se han mantenido y articulado en el tiempo las movilizaciones. Tales aspectos pueden ser de utilidad para cons- truir bases de datos que puedan utilizarse para diversas investigaciones y contribuir a despejar diferentes incógnitas de la acción colectiva y las protestas.

Para ello, se ha estructurado en este artículo una línea argumentativa en el siguiente orden: a. descripción de los principales abordajes teóricos en el estudio de la acción colectiva y las protestas, b. problemas metodológicos más frecuentes en el estudio de las protestas, c. los estudios sobre las protestas en Venezuela, d. propuesta metodológica.

\section{APROXIMACIONES TEÓRICAS AL ESTUDIO DE LA ACCIÓN COLECTIVA Y LAS PROTESTAS}

La producción sobre las aproximaciones teóricas para explicar la acción colectiva ha sido prolifera (Urreiztieta, 2008; Aguiar, 2007; López, 1996, entre otros) y pone de manifiesto la dificultad de adoptar una definición univoca. Así que más que enumerar en este trabajo las principales propuestas teóricas, se pretende tras enunciar sus postulados principales, identificar los rasgos fundamentales que distinguen una acción colectiva que pueda servir de base para su abordaje metodológico y desarrollar futuras hipótesis sobre el tema.

Hay puntos comunes entre los diferentes abordajes, uno de ellos es el relacionado con la relación entre el momento de la decisión individual y su paso a la acción colectiva (Tilly, 1990), lo cual es todavía una dimensión no resuelta. Otro punto común lo constituye el debate sobre la causa y las circunstancias en la que surge la acción colectiva. Responder a las preguntas ¿Cuáles son las razones por las cuales la gente protesta? ¿por qué expresan su descontento a través de acciones de protestas? ¿Bajo qué circunstancia los diferentes actores o individualidades se convierten en un conglomerado para protestas?, constituyen un conjunto de preguntas claves que han intentado responder los analistas de diferentes disciplinas y enfoques. Las respuestas apuntan a relaciones causales o estructurales de la sociedad, así como respuestas relacionadas con las características

\section{$138 \underset{\text { Cumbres }}{\text { REVISTCCINTTICACA }}$}


del conflicto.

A partir de los años 80, surgen preguntas sobre las motivaciones cognoscitivas y emocionales de la acción colectiva, así como sobre los aspectos culturales y de constitución de la identidad colectiva, sobre los usos del lenguaje y los elementos de la estructura social y política presentes para que se desarrolle la acción colectiva.

El carácter dinámico, histórico, intencional y que las acciones se enmarcan en la lógica de las demandas son otros rasgos comunes a las propuestas sobre la acción colectiva. Finalmente, es importante señalar, que el impacto logrado por las acciones colectivas también es un área de interés, menos explorado.

A continuación, se presenta de manera resumida las bondades y debilidades de los principales abordajes teóricos. En este primer grupo de propuestas se encuentran las de énfasis en la decisión de los individuos para actuar ante situaciones de injusticia, indignación o maltrato. Bajo este paradigma funcionalista se intenta responder a la pregunta: ¿cuál es la motivación que hace reaccionar a las personas? Las respuestas van desde las aproximaciones psicológicas con énfasis en los aspectos irracionales, hasta las explicaciones economicistas con énfasis en los aspectos racionales de la acción colectiva.

\subsection{Teorías del comportamiento colectivo (Co- llective behaviour)}

Para las teorías del comportamiento colectivo son las expectativas frustradas de las personas lo que subyace a la acción colectiva, son los sentimientos los detonadores de la movilización por la no satisfacción de sus aspiraciones.

Son los trabajos de Ted Gurr, Why men rebel (1970) pioneros en los enfoques psicosociológicos. Gurr parte del concepto de frustración relativa, entendido como "el estado de tensión, una satisfacción esperada y denegada, generadora de un potencial de insatisfacción y violencia". Además de los aspectos psicológicos, Gurr considera en su a bordaje aspectos culturales y de memoria colectiva, identificando las condiciones sociales que generan frustración y motivan su expresión. Por otra parte, su preocupación por las condicio- nes que originan la violencia, la dimensión simbólica de la protesta y el papel de los medios de comunicación, son elementos novedosos en su aproximación.

Otro representante del paradigma estructural-funcional es Smelser (1962), en este resulta importante el carácter patológico de la movilización, quienes tienen el objetivo de accionar el cambio social en virtud de una cierta racionalidad. Resulta central el concepto de creencia generalizada, que incluye las representaciones, ideologías y creencias como factores generadores de la movilización y no solo los aspectos de orden psicológico.

\subsection{La elección racional}

Este es el enfoque más influyente de los años 70. The logic of the collective action, de Mancur Olson (1966) es la obra más representativa de esta aproximación. Ante la interrogante ¿Cómo implicar a los individuos en actividades colectivas si estos solo se mueven por intereses y beneficios individuales? Se propone una explicación económica del comportamiento colectivo, suponiendo la existencia de intereses individuales y mezquinos, que impiden la acción colectiva en función del bien común.

Por el énfasis en estas variables de tipo individual, Olson no logra presentar una explicación de la acción colectiva que supere las variables motivacionales individuales. La lógica del costo-beneficio que caracteriza este enfoque, deja sin respuestas, aspectos relacionados con la existencia del compromiso compartido, los intereses de grupo y la permanencia en el tiempo.

\subsection{La movilización de recursos}

John McCarthy y Mayer Zald (1973, 1977), señalaron como fundamental la disponibilidad de recursos en la sociedad como elemento necesario para la movilización y la acción colectiva, ya que estos garantizan la posibilidad de organización del movimiento. Esta aproximación da un paso más, al incorporar a su análisis de predominancia económico, nuevas categorías que dan respuesta a los planteamientos no resueltos por Olson, introduciendo el concepto de miembro, que 
designa a las personas y organizaciones que se "adhieren" a la causa.

Neveu (2000) sintetiza en cuatro los aportes de la movilización de recursos; 1 . redefinición de las fronteras de la acción colectiva; 2 . se pregunta cómo se desarrollan las movilizaciones, porque tienen éxito o fracasan, dando explicaciones más allá de las motivaciones; 3 . considera la movilización como un proceso dinámico, que construye una relación de fuerza y sentido, estas relaciones son construcciones sociales y no simples datos; 4 . intenta superar el reduccionismo económico de Olson, e incluye diferentes aspectos de la problemática social que estimulan la movilización.

En esta misma línea explicativa destacan los trabajos de Oberschall (1973). Hace énfasis este autor en aspectos sociales como la intensidad y la naturaleza de los lazos que asocian entre sí a los miembros de un grupo y los vínculos de estos grupos con las diversas autoridades sociales.

Una segunda categoría de propuestas teóricas las encontramos en los enfoques que privilegian la dimensión construccionista de lo colectivo y la dimensión política de la acción colectiva.

\subsection{El análisis de los procesos políticos}

Después de los trabajos de Oberschall, John McCarthy y Mayer Zald, el tema de la organización resulta relevante. La mayor contribución la hace Tilly (1978) al analizar las condiciones sociales de la movilización, vinculando su explicación con la dimensión política de las sociabilidades, situándola en el centro de su propuesta. A partir de aquí, establece que un grupo estará mejor organizado para la defensa de lo que percibe son sus intereses, en la medida que sus redes de sociabilidad voluntarias permitan construir identidades en función de condiciones objetivas.

Por otro lado, Tilly (1990) considera contextos temporales más amplios, para examinar la acción colectiva desde una perspectiva histórica. Considera aspectos como las estrategias, el contexto político, la construcción de sociabilidad y de marcos culturales de actores y movimientos en perspectiva histórica, dando un paso adelante en las explicaciones de acciones colectivas esporádicas en función de incentivos generalmente eco- nómicos.

Esta dimensión política incluida, caracterizada por la relación oportunidad-amenaza de quienes actúan contra la permisividad-represión como respuesta del Estado, da lugar a un enfoque más político de los movimientos sociales, que considera la estructura de oportunidad política eje central de la explicación, desarrollada posteriormente con más fuerza por Tarrow (2004). Se propone entonces, un modelo de interacción racional, que conjuga la teoría de juegos y los modelos de ecuaciones simultaneas de procesos de influencia mutua, incluyendo el análisis estructural que explica los cambios a nivel de actores, identidades y reglas de juego.

\subsection{Los nuevos movimientos sociales}

Boaventura de Sousa (2001), Melucci (1991), Touraine (1990), entre otros autores, han señalado algunos aspectos que distinguen los nuevos movimientos sociales: la adopción de formas orgánicas y nuevos repertorios de acción colectiva, énfasis en las formas de organización (descentralizadas con mayor nivel de autonomía de sus miembros), adoptan una topología menos convencional que las formas clásicas, incluyendo una gama más amplia de acciones donde el papel de los medios de comunicación es fundamental, incluye un nuevo repertorio de reivindicaciones de tipo cualitativo, que surgen de los nuevos temas que han pasado de lo privado a lo público, como opciones sexuales y demandas en derechos humanos.

Aunque la dimensión política forma parte del análisis, el énfasis se sitúa en las condiciones estructurales como determinantes de la acción colectiva. Sin embargo, también han señalado algunas incógnitas en relación a las razones que inducen a los individuos a la movilización.

\subsection{La dimensión cultural de la acción colec- tiva}

Desarrollos posteriores de Mellucci ponen un mayor acento en los procesos de reconocimiento y configuración de identidades de los actores, como factor decisivo en la construcción social de la acción colectiva. Esta nueva visión supone

\section{$140 \quad$ Cumbres}


considerar a los movimientos sociales como generadores de códigos culturales alternativos y da pie a una visión constructivista para el estudio de la acción colectiva. Se separa así de autores del enfoque del proceso político como Tilly, Tarrow, McAdam y Kriesi, quien ponen el acento en sus abordajes en la confrontación de las acciones colectivas con las autoridades.

Melluci entonces propone entender la acción colectiva en un marco de relaciones sociales con límites y posibilidades para los actores individuales y grupales, en un proceso de negociación de intereses opuestos hasta construir un nosotros, dándole identidad a la acción. Sin embargo, esos límites que impone el contexto no permiten a los actores transformarlo. En resumen, hay un nuevo acercamiento al tema en cuestión, que implica examinar el proceso de configuración de lo colectivo, con un acercamiento cualitativo en relación a los actores en un tiempo y espacio determinados.

\subsection{La estructura de las oportunidades políti- cas}

En los años 70, Meter Eisinger y Michael Lipsky intentaron identificar las condiciones que favorecen la movilización y las protestas, las variaciones en su expresión, en función de los rasgos receptivos del sistema político ante las demandas ciudadanas. Propusieron para esto la estructura de oportunidades políticas, definida como el grado de probabilidad que tienen los grupos de acceder al poder e influir en el sistema político.

A partir de entonces autores como Tarrow, Tilly, McAdam, Jenkis y Perrow suscribieron esta propuesta. Pero a la vez comenzaron a señalar limitaciones del modelo, entre estos tenemos la dificultad de la delimitación o no de las oportunidades en términos políticos y dudas sobre la flexibilidad de los conceptos y aplicabilidad al tratar de explicarlo todo.

En términos generales, la estructura de oportunidades políticas, hace referencia a las condiciones políticas que favorecen o inhiben la acción colectiva. Tarrow (2004) propone el análisis en función de cinco categorías básicas: 1. el grado de apertura/cierre de acceso político formal de nuevos actores, 2. el grado de estabilidad/inestabilidad de las preferencias políticas, 3. la disponibilidad y posición estratégica de los potenciales socios o aliados, 4 . los conflictos políticos o emergencias de divisiones en el seno de las elites y 5 . la disminución de la capacidad (o voluntad) del Estado para reprimir la disidencia.

\subsection{La estructura del contexto}

Rucht (1999) propone la importancia del estudio del contexto para el entender el surgimiento y evolución de las estructuras de un movimiento. Por estructura del contexto se entiende el conjunto de factores del entorno de un movimiento que facilitan o limitan la construcción de una estructura específica, los recursos de que dispone y la viabilidad para concretar acciones de protesta. Aclara que, si bien el contexto político es importante, aunque insuficiente, existen otros elementos constitutivos del entorno como los sociales y culturales. En resumen, la propuesta de Rucht se propone ampliar el concepto de oportunidades políticas al incorporar las dimensiones del contexto cultural, social además del político. Para esto sugiere, además, la estrategia de estudios comparados entre movimientos, que permita cruzar información tanto de la dinámica interna del movimiento como de las condiciones externas en distintos escenarios nacionales y en un lapso de tiempo también diferencial (González, 2006).

Desde estas diferentes perspectivas teóricas, nos encontramos varias definiciones de acción colectiva y de protestas, que harán énfasis en distintas dimensiones de un concepto complejo; vale resaltar que la acción colectiva es una categoría de análisis amplia que arropa un conjunto de acciones donde se incluyen las protestas.

Como punto de partida señalaremos la definición de acción colectiva propuesta por Tarrow, en virtud que esta ha sido ambigua y se solapa con otras categorías analíticas como los Movimientos Sociales o Comportamientos Colectivos.

La acción colectiva adopta muchas formas: puede ser breve o mantenida, institucionalizada o subversiva, monótona o dramática. En su mayor parte se produce en el marco de las ins- 
tituciones por parte de grupo constituidos que actúan en nombre de objetivos que difícilmente harían levantar una ceja a alguien. Se convierte en contenciosa cuando es utilizada por gente que carece de acceso regular a las instituciones, que actúan en nombre de reivindicaciones nuevas o no aceptadas y que se conduce de un modo que constituye una amenaza fundamental para otros o las autoridades. (Tarrow, 2004: 24)

Para Gamson (1995) la acción colectiva va más allá de las actividades para la vida diaria o la subsistencia, en pos de acciones que tiendan a cambiar algunas condiciones de nuestras vidas, con las cuales se busca enfrentar una situación social injusta y solitaria. Desde esta perspectiva, la acción colectiva es definida como un ejercicio político y social -con mayores o menores niveles de organización que busca el logro de demandas comunes.

Por otra parte, Mellucci (1996), agrega el componente cultural, al considerar que las acciones colectivas construyen sistemas emergentes de cultura política que se entretejen con la vida diaria, proveen nuevas expresiones de identidad y van en oposición directa al orden dominante.

Como vemos las acciones colectivas son expresiones con una clara dimensión política, ya sea en defensa del orden político o económico establecido, en protesta o en resistencia, u oposición a ese orden.

Pero estos conceptos y otros, denotan la dificultad de lograr un único concepto, que pasa por la dificultad también de definir qué es lo colectivo. En resumen, las tendencias a la definición dependerán de la pregunta de investigación, aquí el cómo, el cuándo y el bajo qué condiciones se produce la acción colectiva, incluyendo las condiciones estructurales y contextuales, serán determinantes para hacer énfasis en las dimensiones señaladas por las diferentes propuestas teóricas. En consecuencia, una propuesta metodológica de registro, debe ser lo suficientemente amplia para poder servir a varios objetivos de investigación o perspectivas de análisis.

En relación al concepto de protesta, Schuster y Pereyra (2001) se esfuerzan por integrar elementos de varias propuestas, así definen la pro- testa social como "los acontecimientos visibles de acción pública contenciosa de un colectivo, orientados al sostenimiento de una demanda (en general, con referencia directa o indirecta al estado)". Esta definición está en línea con lo propuesto por Tarrow (2004), la acción colectiva es realizada por personas que tienen difícil acceso al juego político institucional y que actúa en nombre de reivindicaciones constitutivas de amenaza a otros grupos sociales dominantes o a las autoridades establecidas.

En resumen, la protesta se refiere a acontecimientos visibles que tienen un objetivo de un colectivo sobre una demanda basada y justificada en el reclamo de un derecho. La propuesta para su estudio apunta a analizarla desde un punto de vista interno y otro externo. Adicionalmente, las acciones a favor de políticas públicas, no se consideran acciones contenciosas de protestas y deben quedar fuera de los registros que sobre las mismas se realicen.

\section{LOS ACERCAMIENTOS METODOLÓGICOS}

A diferencia de los numerosos trabajos sobre las propuestas teóricas para el estudio y explicaciones de la acción colectiva y las protestas, las referencias a los aspectos metodológicos son de menor producción. En este sentido, es necesario hacer indicaciones precisas apoyadas en lo conceptual para identificar las dimensiones básicas de análisis, categorías y variables, que nos permita el abordaje de casos concretos.

El asunto es mucho más complejo que el simple registro de la aparición del evento, con información adicional sobre el quien, cuando y donde. Y como quedó evidenciado en la sección anterior, los registros deben apoyarse en propuestas teóricas que permitan articular la compleja dinámica de las acciones sociales, expresar su marco contextual, identificación de los actores, la tipología y objetivo de sus acciones, la posibilidad de identificar los limites, posibilidades de articulación con otras acciones y actores y evaluar su impacto en un tiempo y espacio determinados.

Por otra parte, el tema introduce otras parti-

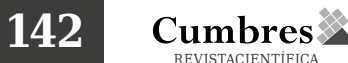


cularidades, 1. generalmente el evento ocurre de manera espontánea, por lo que existe la imposibilidad de registrar en presente e identificar otras dimensiones (agentes organizativos, motivacionales, culturales, entre otros), 2. el evento en apariencia tiene una clara aparición y pareciera un evento aislado, pero en realidad es un proceso, con unos antecedentes y unas consecuencias, 3. el uso de fuentes documentales (caracterizado por el uso de medios de comunicación), la calidad de la información reseñada afecta el dato recogido, pudiendo faltar información, 4 . en consecuencia, puede existir un subregistro de eventos, ya que dependiendo del medio utilizado para el registro y su visibilidad, el mismo puede no estar reseñado en los medios o puede ser reseñado por varios; sin embargo, si el registro incluye una variedad de fuentes, se recoge una tendencia bien cercana a la realidad del fenómeno. Esta postura es sostenida por los trabajos aquí revisados en Argentina, México y Colombia.

Existen otros problemas ligados a la protesta, todavía sin resolver como lo es la magnitud y la contundencia de la acción. En Venezuela, hemos tenido eventos de asistencia masiva, sin el alcance de los objetivos propuestos, mientras otros eventos con menos participación tienen un mayor impacto, en consecuencia, no son equivalentes la fuerza de la acción y sus efectos. Entonces, ¿cómo calcular la asistencia de actores, crear una escala que permita medir la intensidad y la magnitud de la protesta?, son todavía elementos que quedan sin respuesta.

Otros elementos de orden metodológicos para superar las debilidades de los registros, implican tomar ciertos conocimientos de Estadísticas para la correcta definición de las variables y los valores que estas adopten. Generalmente estamos trabajando con variables de tipo cualitativo nominal, que deben estar claramente definidas, según las indicaciones teóricas ya señaladas y que cumplan con los requisitos de exhaustividad y exclusión para sus registros. Estos requisitos en algunos casos no se cumplen en los datos obtenidos, introduciendo errores en las futuras codificaciones y conclusiones (Bohrnstedt, 1994).

En síntesis, la investigación teórica y metodo- lógica de la acción colectiva y la protesta supone un gran desafío que implica proponer un esquema que articule las dimensiones metodológicas con una base conceptual que subyace al tema tratado, que si bien no pretende ser un esquema modelo para el registro de la acción colectiva y la protesta, puede servir de orientación a las personas u organizaciones que recogen información, que alimentan líneas de investigación que intentan responder aspectos de la acción colectiva y la protesta, de manera que sus esfuerzos puedan ser más fructíferos y de más largo alcance en la información suministrada y que el registro obtenido se acerque mucho más a la realidad.

\section{LOS TRABAJOS SOBRE PROTESTAS}

Los estudios en América Latina que abordan el tema de la protesta también utilizan como fuente primaria la revisión documental y periodística. Muy importante son los trabajos de Inigo (2001) sobre los cierres de vías, y Scribano (2003), en Argentina, en especial el segundo autor presenta además información sobre las protestas en Brasil, Chile, Uruguay y Paraguay. Los trabajos de Rodríguez (2005) en México, y en Colombia sobre la acción colectiva y las acciones violentas de Barreto (2007) y Sabucedo (2006).

En Venezuela los trabajos de Margarita López Maya son pioneros en el estudio de la protesta, apellidada popular en sus primeras aproximaciones. En relación al aspecto aquí abordado, la autora presenta en el año 1996 unas notas teórico-metodológicas sobre la protesta popular venezolana. Señala para la fecha el poco interés suscitado por el tema, conducen a hipótesis de carácter provisorio. Siguiendo los principales acercamientos teóricos, fundamentalmente desde la historia y sociología, propone dos coordenadas de análisis. La primera, las distintas modalidades de protestas según un criterio cronológico, y la segunda, según su composición social y objetivos.

En relación a la primera coordenada, propone el estudio de las protestas en tres tiempos, 1. desde principio del siglo XX hasta mediados de 
los años 40, 2. desde los años 40, hasta finales de la década de los años 60, a partir de los años 80 hasta la fecha de su artículo.

En la segunda coordenada, apoyada en la propuesta de Rudé y respondiendo a la pregunta ¿Quién protesta?, propone un primer grupo, de los sectores sociales que "galopan entre dos mundos", los pobres y marginales, tanto campesino como urbanos, que protestan a favor o en contra de un orden establecido, siendo su protesta "poco moderna" en términos de su organización, dirección y objetivos. El segundo grupo estará conformado por la protesta moderna propiamente dicha, incluye aquí a las acciones del sector laboral formal e informal, público o privado, es decir, obreros y empleados. El tercer grupo, corresponde a la protesta estudiantil, quienes poseen una composición social y reivindicaciones modernas, pero sin articularse a la economía formal.

Este trabajo pionero, no discute el problema de la fuente para la recolección de información, asomando la dificultad de utilizarlas como fuente primaria.

Trabajos más recientes sobre la protesta popular en Venezuela de la misma autora (2003, 2004), introducen cambios conceptuales. El primero de ellos es asumir otras aproximaciones teóricas, moviéndose de posturas marxista, gramsciana, Tarrow, Tilly, hacia aproximaciones más recientemente como los marcos de acción colectiva usado en primer lugar por Goffman (1974).

En relación a los aspectos metodológicos para la construcción de la base de datos "El Bravo pueblo", de la cual fue directora, se utilizaron las reseñas de el diario El Nacional, y posteriormente incorporan los informes anuales de Provea, quienes utilizan para sus reportan un número mayor de diarios tanto de circulación nacional como regionales. Otros trabajos han incluido la recolección de reportes, registro de eventos simbólicos (como volantes, pancartas y consignas) y entrevistas estructuradas, en especial para los trabajos que abordan valores, símbolos y los elementos culturales de la movilización.

También encontramos los trabajos de tesis de Salamanca (2004) y Débora Calderón (2010).
El primero, revisa la ola de protestas del periodo postgomescista (1935-1937) a través del enfoque de procesos político, cambiando el foco de atención de las explicaciones partido-céntricas a otra más socio-céntrica. Para la recolección de información se vale de varias fuentes como documentación primaria, referencias bibliográficas y narrativas históricas, que le dan solidez a la investigación.

El trabajo de Calderón, aborda las protestas del año 2007, utilizando los datos Provea, aplicando análisis estadísticos como el Análisis de Correspondencias Binarias, Análisis de Correspondencias Múltiples y el Análisis de Clasificación, sin modificación de los datos originales. Los resultados más importantes obtenidos en la investigación, indican que las manifestaciones públicas del 2007 pueden ser explicadas a través de dos factores fundamentales: la organización de las manifestaciones públicas, es decir, su preparación logística; y el componente político de las mismas según las demandas realizadas.

\section{LA PROPUESTA METODOLÓGICA}

Con el objetivo de diseñar una ruta metodológica con indicaciones precisas teóricas que permita generar unidades de registro, que den cuenta de categorías y variables se propone una estrategia mixta de fuentes de información orientada a captar la protesta, en una dimensión espacio-tiempo y su forma de expresión que implicaría:

a. Revisión y análisis de archivos periodísticos de prensa (en papel y digital) y TV, para obtener una cobertura nacional y regional.

b. Testimonios de los actores, además de registrar las declaraciones de los medios de comunicación, es necesario incluir entrevistas breves o relatos de los actores involucrados.

c. La información obtenida debe generar una base de datos amplia que conlleven una descripción de los eventos de protestas presentados en un documento con varias celdas de registro a saber:

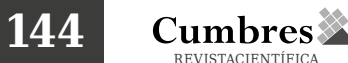


1) IDENTIFICACIÓN DE LA PROTESTA. Presentar información sobre la protesta, lugar de ocurrencia, fecha, y fuente del registro.

2) EN RELACIÓN A LOS ACTORES INVOLUCRADOS. Quien protesta y contra quien se protesta. Tomando en consideración los trabajos realizados con el caso venezolano reciente encontramos:

- Estudiantes

- Trabajadores

- Desempleados

- Transportistas y Motorizados

- Familiares de víctimas de delincuencia

- Vecinos (por definir)

- Usuarios de centros de salud

- Jubilados y pensionados

- Indígenas

- Campesinos

- Comerciantes

- Reclusos

- Padres y Representantes

- Discapacitados

- Mujeres

- Adultos mayores

- Profesionales (Periodistas, farmaceutas, médicos, profesores)

Si fuera relevante políticamente, debido al contexto ya enunciado con anterioridad, puede resultar importante identificar si estos actores son afectos al gobierno o no.

3) EN RELACION A LA ACCION. Métodos que se utilizan para conseguir la reivindicación, tipo de acción utilizada para el logro de un objetivo (tipo de protesta).

- Cierre de calle

- Concentraciones

- Marchas

- Toma de establecimientos, secuestros

- Paros laborales

- Vigilias

- Caravanas

- Cacerolazos

- Huelga de hambre

- Pintas en la calle (grafitis) y Entrega de volantes

- Pupitrazos
- Otros

4) OTROS DATOS: acciones violentas en las manifestaciones, represión, lesionados, heridos y muertes también son importantes y/o destrozos a propiedades y objetos.

5) EN RELACION A LAS DEMANDAS. Es fundamental el registro de la acción que impulsa la acción, muy ligado a las demandas por Derechos Humanos. Estos son de tipo:

- Laborales

- Educativos

- Salud

- Sociales

- Civiles y políticos

- Vivienda

- Derecho a la justicia

- Servicios Públicos

- Otros

6) EN RELACION A LA IDENTIDAD. Un aspecto más difícil de registrar corresponde a la identificar valores, creencias y otros elementos vinculantes que hacen coincidir a las personas en el alcance de un objetivo común., especialmente cuando el registro se hace una vez acontecido el hecho.

7) VARIABLES DE ORDEN POLITICO. Finalmente, existen otras variables de orden político como la presencia de organizaciones partidistas o gremiales, acceso o no al sistema político, liderazgos o voceros de grupos, presencia o respuesta por parte de representante del Estado que terminan por configurar las dimensiones de la acción.

8) ARTICULACION DE LOS HECHOS Y ACTORES. En la lectura que sobre el registro se realice debe existir la posibilidad de discriminar la articulación de actores o acciones o si bien, estas son episódicas-únicas.

Como punto de cierre, podemos señalar que esta propuesta responde a la necesidad de definir dimensiones de registro y análisis sobre la base de propuestas teóricas e investigativas que permitan ofrecer datos para atender preguntas de 
diversa índole en relación a la acción colectiva y la protesta.

\section{REFERENCIAS BIBLIOGRÁFICAS}

Aguiar, F. (2007). Siete tesis sobre racionalidad, identidad y acción colectiva. Revista Internacional de Sociología, 45(46), 63-86.

Barreto, I. (2007). Violencia política: algunas consideraciones desde la psicología social. Revista Diversitas, 3(1), 109-119.

Boaventura de Sousa, S. (2001). Los nuevos movimientos sociales. OSAL, (5), 177-188.

Bohrnstedt, G. (1994). Statistics for social data analysis. Illinois: F.R. Peacock Publishers, INC.

Calderón, D. (2010). Caracterización y clasificación de las manifestaciones públicas en Venezuela registradas en el 2007 (Trabajo especial de grado para optar al título de Especialista en Análisis de Datos en Ciencias Sociales). UCV, Caracas.

Gamson, W. (1995). Constructing Social Protest. En H. Johnston y B. Klandermans (Eds.), Social movements and culture (pp. 85-106). Minneapolis: University of Minnesota Press.

Goffman, E. (1974). Frame analysis. Boston: Northeastern University Press.

Gurr, T. (1970). Why men rebel. Princeton, NJ: Princeton University Press.

Iñigo, N., \& Cotarelo, M. (2001). Hito en el proceso de luchas populares. En J. Seoane, Movimientos sociales y conflictos en América Latina (1-138). Buenos Aires: CLACSO. Recuperado de http://bibliotecavirtual.clacso.org. ar/ar/libros/osal/seoane/inigo.rtf

López, M. (1996). Notas teórico-metodológicas para la investigación: la protesta popular en la Venezuela contemporánea. Revista Venezolana de Análisis de Coyuntura, 2(2), 59-71.

López, M. (2003). La protesta popular venezolana entonces y ahora: ¿́cambios en la política de calle?. Revista Politeia, (30), 157-181.

López, M. (2003). Movilización, institucionalidad y legitimidad en Venezuela. Revista Venezolana de Economía y Ciencias Sociales, 9(1), 211226.

López, M. (2004). Venezuela 2001-2004: Actores y estrategias. Cuadernos del Cendes, (56), 105128.

López, M. (2006). Novedades y continuidades de la protesta popular en Venezuela. Revista Venezolana de Economía y Ciencias Sociales, 12(1), 11-30.

Melucci, A. (1991). ¿Qué hay de nuevo en los nuevos movimientos sociales?. En E. Laraña y J. Gusfield (Eds.), Los nuevos movimientos sociales: De la ideología a la identidad (119-150). Madrid: Centro de Investigaciones Sociológicas.

Melucci, A. (1996). The process of collective identity. En A. Melucci, Challenging codes: Collective action in information age (pp. 41-63). Cambridge: Cambridge University Press.

Neveu, E. (2000). Sociología de los movimientos sociales. Barcelona: Editorial Hacer.

Oberschall, A. (1973). Social conflicto and social movements. Englewood Cliffs, New Jersey: Prentice-Hall.

Olson, M. (1966). La Lógica de la Acción Colectiva. En M. Olson, Diez textos básicos de ciencia política (pp. 203 - 220). Madrid: Ariel.

Rodríguez, G. (2005). La protesta política: una propuesta para su estudio. Espacios Públicos,

\section{Cumbres}


8(16), 48-59.

Rucht, D. (1999). El impacto de los contextos nacionales sobre la estructura de los movimientos sociales: Un estudio comparado transnacional y entre movimientos. En D. McAdam, J. McCarthy, \& M. Zald (Eds.), Movimientos sociales: perspectivas comparadas (262-287). Madrid, Ediciones Istmo.

Sabucedo, J. (2006). Legitimación de la violencia y contexto. Estudios de Psicología, 27(3), 279291.

Salamanca, L. (2004). Protesta y política en la Venezuela contemporánea 1935-1937. (Tesis doctoral). UCV, Caracas.

Schuster, F., \& Pereyra, S. (2001). La protesta social en la Argentina democrática. Balance y perspectivas de una forma de acción política. En N. Giarracca, La protesta social en la Argentina. Buenos Aires: Alianza.

Scribano, A. (2003). Reflexiones sobre una estrategia metodológica para el análisis de las protestas sociales. Sociologías, Porto Alegre, (9), 64-104.

Smelser, N. (1962). Theory of collective behaviour. Londres: Routledge \& Kegan Paul

Tarrow, S. (2004). El poder en movimiento, los movimientos sociales, la acción colectiva y la política. Madrid: Alianza Editorial.

Tilly, C. (1978). From Mobilization to Revolution. USA: Addison-Wesley.

Tilly, C. (1990). Modelos y realidades de la acción colectiva popular. Zona abierta, 54/55, 167195.

Touraine, A. (1990). Movimientos sociales de hoy: Actores y analistas. Barcelona: Editorial Hacer.
Urreiztieta, M. (2008). La comprensión por el contexto: Los movimientos sociales y los contextos de la acción colectiva. Espacio Abierto Cuaderno venezolano de Sociología, 17(1), 87108.

Zald, M. (1977). Recourse mobilization and social movement: A partial theory. The American Journal of Sociology, 82(6), 1212-1241. 